\title{
ATRIBUTOS FÍSICOS E QUÍMICOS DE UM LATOSSOLO, SOB DIFERENTES SISTEMAS DE CULTIVO
}

\author{
Physical and chemical attributes of a typical Acrustox soil \\ under different systems of cultivation
}

\author{
William Diniz Bilibio', Gilberto Fernandes Corrêa², Elias Nascentes Borges²
}

\begin{abstract}
Resumo
Diferentes sistemas de manejo impactam em intensidades diferentes os atributos físicos e químicos do solo. Com o propósito de avaliar alterações em propriedades físicas e químicas de um Latossolo Vermelho distrófico, conduziu-se um experimento na Fazenda Glória/UFU, desde o ano 2000. O latossolo foi disposto num esquema de delineamento em blocos casualizados e conduzido durante sete anos para avaliar algumas alterações em atributos físicos e químicos do solo, nas profundidades de 0-5; 5-15 e 15-30 cm. Os sistemas de manejo avaliados foram o convencional e os considerados conservacionistas. Os parâmetros analisados foram: densidade do solo, porosidade total, macro e microporosidade, estabilidade de agregados, carbono orgânico total (COT), fósforo (P), cálcio $\left(\mathrm{Ca}^{2+}\right)$, magnésio $\left(\mathrm{Mg}^{2+}\right)$, potássio $\left(\mathrm{K}^{+}\right)$, nitrogênio $(\mathrm{N})$ e alumínio $\left(\mathrm{Al}^{3+}\right)$. Foram verificadas diferenças estatísticas entre os sistemas de manejo conservacionistas e o convencional. Os resultados indicam aumento da densidade do solo com a profundidade, associada à redução da porosidade total e da relação macro/microporos, bem como uma menor estabilidade de agregados no sistema convencional, em relação aos sistemas conservacionistas. Para os atributos químicos do solo, diferenças estatísticas entre os tratamentos conservacionistas e convencional foram observadas apenas nos primeiros $5 \mathrm{~cm}$ do solo, para os elementos P, Ca e K. A correção do $\mathrm{Al}$ é mais eficiente no sistema convencional, devido à incorporação do calcário ao solo, independente da aplicação de gesso agrícola.
\end{abstract}

Termos para indexação: Sistemas de manejo de solo, gesso agrícola, solo de cerrado, PD.

\section{ABSTRACT}

Different management systems impact soil physical and chemical characteristics differently. Physical and chemical attribute changes of a typical Acrustox soil submitted to different soil preparation systems were evaluated in an experiment at the Glória/UFU farm since the year 2000. The experimental design was randomized blocks, and the experiment was conducted for seven years to evaluate some physical and chemical attributes of the soil, at the depths of 0-5; 5-15 and 15-30 cm, with different management systems of handling (conventional and conservation). The following parameters were analyzed: density, total porosity, macro and micro porosity, stability of aggregate, total organic carbon (TOC), phosphorus $(\mathrm{P})$, calcium $\left(\mathrm{Ca}^{2+}\right)$, magnesium $\left(\mathrm{Mg}^{2+}\right)$, potassium $\left(\mathrm{K}^{+}\right)$, nitrogen $(\mathrm{N})$ and aluminum $\left(\mathrm{Al}^{3+}\right)$. Statistical differences between the two management systems of handling, conservational and conventional, wasStatistical differences between the two management systems of handling, conservational and conventional, were observed. Increased density with the depth, associated with reduction of the total porosity and the relation macro/micropores as well as a lesser lesser aggregate stability of aggregate in the conventional system, was observed in relation to the conservation systems. Significant differences for the soil chemical attributes of the soil between the conservation and conventional systems, were observed only in the top $5 \mathrm{~cm}$ of the soil, and only for $\mathrm{P}, \mathrm{Ca}$ and $\mathrm{K}$. Aluminum correction was more efficient in the conventional system, due to incorporation of lime to the soil, independently of the application of gypsum.

Index terms: Soil management, management systems, calcium sulfate, cerrado soils.

\section{(Recebido em 16 de junho de 2009 e aprovado em 23 de março de 2010)}

\section{INTRODUÇÃO}

Atualmente, vários sistemas de manejo têm sido adotados na agropecuária brasileira. Caberia à pesquisa propô-los, mas particularmente em se tratando de sistemas de manejo, esses em geral têm sido desenvolvidos por agricultores. A pesquisa tem desempenhado mais o papel de avaliá-los, tendo em vista o uso sustentável dos solos. Essa avaliação requer o estudo, através de anos sucessivos, dos efeitos sobre as propriedades físicas, químicas e biológicas do solo.
No Brasil, grandes áreas, especialmente sob pastagens degradadas, necessitam ser reintegradas ao sistema produtivo, num processo de mitigação da abertura de novas áreas. À medida que o solo vai sendo submetido a usos agrícola e/ou pecuário, as propriedades físicas sofrem alterações geralmente desfavoráveis ao desenvolvimento vegetal (Anjos et al, 1994; Andreola et al., 2000; Albuquerque et al., 2001). O uso do solo leva ao comprometimento de sua estrutura através da compactação, afetando a relação macro/microporos e,

\footnotetext{
'Universidade Federal de Uderlândia/UFU - Instituto de Ciências Agrárias/ICIAG - Uberlândia, MG - williambilibio@hotmail.com TUniversidade Federal de Uderlândia/UFU - Instituto de Ciências Agrárias/ICIAG - Uberlândia, MG
2Universidade Federal de Uderlândia/UFU - Instituto de Ciências Agrárias/ICIAG - Uberlândia, MG
} 
consequentemente, a dinâmica do ar e da água, havendo também danos à atividade biológica Sá (1993), Maria et al. (1999) e Silveira \& Stone (2001). Do ponto de vista químico, de um modo geral há que se considerar a textura e o teor de matéria orgânica por estarem estreitamente relacionados à capacidade de troca de cátions (CTC). Em solos com maiores teores de matéria orgânica e/ou mais argilosos, a capacidade tampão é substancialmente maior e, consequentemente, o solo comporta-se como um sistema mais conservador. Isso facilita a construção da fertilidade do solo e sua manutenção. Por outro lado, pode aumentar o risco de desequilíbrios nutricionais mediante adubações desbalanceadas, uma vez ultrapassada a capacidade tampão do solo (Corrêa, 1984). Nesse aspecto, os sistemas conservacionistas, como o plantio direto, tendem a concentrar os nutrientes nos primeiros centímetros do solo, favorecendo maior aporte de nutrientes superficialmente, podendo gerar desequilíbrios nutricionais.

Obejtivou-se, neste trabalho, avaliar o efeito dos sistemas de manejo convencional e conservacionista sobre alguns atributos físicos e químicos do solo, após sete anos de implantação.

\section{MATERIAL E MÉTODOS}

O experimento de campo foi instalado no ano de 2000 na Fazenda Glória, Município de Uberlândia-MG, num Latossolo Vermelho distrófico (LVd) fase cerrado, anteriormente ocupado com pastagem de braquiária. Pela classificação de Köpen, o tipo climático da área é Cwa, mesotérmico (Empresa Brasileira de Pesquisa AgropecuáriaEmbrapa, 1982). As parcelas experimentais, com área de 275 $\mathrm{m}^{2},(11 \times 25 \mathrm{~m})$, foram dispostas em um delineamento de blocos casualizados com quatro repetições. Antes da instalação dos tratamentos, o solo foi analisado para obter-se os dados de referência, apresentados na Tabela 1 .

Desde o início da pesquisa, foi estabelecida a sucessão anual das culturas de milho e soja tendo a Brachiaria decumbens como cobertura de entressafra, nos sistemas de manejo ditos conservacionistas. Todas as parcelas receberam, anualmente (no mês de junho), calcário dolomítico na dosagem necessária para atingir saturação por bases de $50 \%$. Nas parcelas que receberam gesso como tratamento, esse foi aplicado com o calcário, levandose em conta a textura do solo, conforme consta do boletim da Comissão de Fertilidade do Solo do estado de Minas Gerais-CFSEMG (1999).

O experimento compreendeu os seguintes tratamentos: sistema de cultivo convencional com calcário + gesso agrícola, incorporados com grade aradora (CCCG); sistema de cultivo convencional somente com calcário, incorporado com grade aradora (CCC); sistema de cultivo com ausência total de preparo do solo com calcário + gesso agrícola aplicados na superfície (sem incorporação) (APCG); sistema de cultivo mínimo com calcário parcialmente incorporado com o arado escarificador (CMC); sistema de cultivo mínimo com calcário + gesso agrícola, parcialmente incorporados com arado escarificador (CMCG); sistema de cultivo com total ausência de preparo do solo, somente com calcário aplicado na superfície (sem incorporação) (APC); sistema de cultivo em plantio direto com calcário + gesso agrícola, porém incorporados com grade aradora apenas na implantação do experimento, no ano agrícola de 2000 (PDCG) e aplicados em superfície em anos subseqüentes, quando a saturação por bases foi menor que $50 \%$. As adubações nos cultivos de milho e soja (rotação) foram realizadas de acordo com recomendações da CFSEMG (1999).

Em $1^{\circ}$ e 2 de junho de 2007, após a colheita do milho, foram retiradas amostras com auxílio de enxadão e do anel de Koppeck. Assim, amostras deformadas e indeformadas de solos foram coletadas nas profundidades de 0-5, 5-15 e 15-30 cm, em cada tratamento. Essas foram submetidas às análises de densidade do solo, porosidade total, macro e microporosidade, estabilidade de agregados, fósforo, cálcio, magnésio, potássio e alumínio, conforme Embrapa (1997) e COT conforme Yeomans \& Bremner (1988), N conforme Tedesco et al. (1985). As análises estatísticas constaram do teste de Tukey, a 0,05 de significância, processadas com o programa estatístico SISVAR (Ferreira, 2000).

Tabela 1 - Caracterização química e física do solo (LVd), profundidade de 0-20 cm, realizada em junho de 2000

\begin{tabular}{|c|c|c|c|c|c|c|c|c|c|c|c|c|}
\hline $\mathrm{pH}$ & $\mathrm{P}$ & $\mathrm{K}$ & $\mathrm{Ca}$ & $\mathrm{Mg}$ & $\mathrm{Al}$ & $\mathrm{H}+\mathrm{Al}$ & $\mathrm{V}$ & M.O. & Ds & Areia & Silte & Argila \\
\hline água & \multicolumn{2}{|c|}{$\mathrm{mg} \mathrm{dm}^{-3}$} & & - & ${ }_{c} \mathrm{dm}$ & & & $\%$ & $\mathrm{~kg} \mathrm{dm}^{-3}$ & & $\mathrm{~g} \mathrm{~kg}^{-}$ & \\
\hline 5,2 & 1,0 & 31 & 0,2 & 0,1 & 0,6 & 4,0 & 8 & 2,1 & 1,22 & 550 & 30 & 420 \\
\hline
\end{tabular}

pH em água 2,5:1, Ca, Mg, Al- (Embrapa, 1997); K , P disponível (extrator Mehlich 1) (Embrapa, 1997); C orgânico total (COT) (Yeomans \& Bremner, 1988); H+Al - método de determinação Ca(OAc) 2 pH 7,5 (Embrapa, 1997); V\% calculado pela (soma de

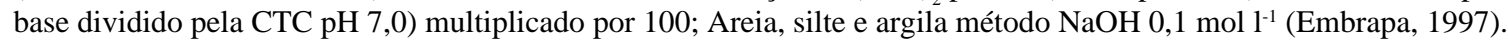




\section{RESULTADOS E DISCUSSÃO}

Os resultados analíticos, no que se refere a atributos físicos (Tabela 2), permitem destacar algumas diferenças, notadamente quando se compara sistema convencional com sistemas conservacionistas. Observase que a densidade do solo, na profundidade $0-5 \mathrm{~cm}$, não apresentou diferenças significativas entre os tratamentos e foram menores devido à maior presença de matéria orgânica nos primeiros centímetros do solo. Uma análise de conjunto indica que houve compactação em todos os sistemas de cultivo, comparando a densidade de caracterização do solo antes da instalação do experimento $\left(2,22 \mathrm{~kg} \mathrm{dm}^{3}\right.$ - Tabela 1) com aquelas determinadas em 2007 (Tabela 2), nos diversos tratamentos (sistemas de cultivo).

Observa-se também que os diâmetros médio geométrico - DMG diminuíram com a compactação subsuperficial nos sistemas conservacionistas, enquanto nos tratamentos convencionais a diminuição do DMG ocorreu apenas na camada superficial $(0-5 \mathrm{~cm})$, devido à desagregação provocada pelas gradagens anuais. Os primeiros centímetros do solo $(0-5 \mathrm{~cm})$, nos sistemas conservacionistas, apresentaram maior estabilidade de agregados (maior DMG) devido à matéria orgânica e à ausência de revolvimento do solo. Esses resultados são corroborados pela literatura (Miyasaka et al., 1996; Cruz et al., 2003).

Analisando os atributos químicos (Tabela 3), observa-se que os teores de nutrientes acompanham os teores de carbono orgânico total (COT). Eles decrescem com a profundidade, conforme se verifica nos solos, em sistemas naturais, devido à ciclagem de nutrientes promovida pelas plantas. Os dados de COT indicam que os diferentes sistemas de cultivo não alteraram essa tendência.

Quanto aos outros nutrientes (P, Ca, Mg e K), os teores decrescem substancialmente da primeira seção de amostragem $(0-5 \mathrm{~cm})$ para as outras profundidades $(5-15 \mathrm{e}$

Tabela 2 - Valores de densidade, porosidade total, macro e microporos e agregados de um Latossolo sob diferentes sistemas de cultivo, em diferentes profundidades.

\begin{tabular}{ccclcccc}
\hline Prof/Trat & CCCG & CCC & CMCG & CMC & APCG & APC & PDCG \\
\hline $0-5 \mathrm{~cm}$ & $1,28 \mathrm{Ab}$ & $1,21 \mathrm{Ab}$ & $1,35 \mathrm{Ab}$ & $1,26 \mathrm{Aa}$ & $1,35 \mathrm{Ab}$ & $1,33 \mathrm{Aa}$ & $1,34 \mathrm{Aa}$ \\
$5-15 \mathrm{~cm}$ & $1,52 \mathrm{Aa}$ & $1,37 \mathrm{Ba}$ & $1,48 \mathrm{ABa}$ & $1,36 \mathrm{Ba}$ & $1,47 \mathrm{ABa}$ & $1,34 \mathrm{Ba}$ & $1,44 \mathrm{ABa}$ \\
$15-30 \mathrm{~cm}$ & $1,48 \mathrm{Aa}$ & $1,44 \mathrm{Aa}$ & $1,41 \mathrm{Aab}$ & $1,35 \mathrm{Aa}$ & $1,43 \mathrm{Aab}$ & $1,40 \mathrm{Aa}$ & $1,38 \mathrm{Aa}$ \\
\hline Prof/Trat & CCCG & $\mathrm{CCC}$ & CMCG & $\mathrm{CMC}$ & APCG & APC & PDCG \\
\hline $0-5 \mathrm{~cm}$ & $0,47 \mathrm{Aa}$ & $0,48 \mathrm{Aa}$ & $0,41 \mathrm{Ba}$ & $0,43 \mathrm{Ba}$ & $0,40 \mathrm{Ba}$ & $0,41 \mathrm{Ba}$ & $0,40 \mathrm{Ba}$ \\
$5-15 \mathrm{~cm}$ & $0,36 \mathrm{Bb}$ & $0,40 \mathrm{ABb}$ & $0,37 \mathrm{ABb}$ & $0,41 \mathrm{Aa}$ & $0,38 \mathrm{Aba}$ & $0,39 \mathrm{Aba}$ & $0,38 \mathrm{Aba}$ \\
$15-30 \mathrm{~cm}$ & $0,35 \mathrm{Bb}$ & $0,41 \mathrm{Ab}$ & $0,40 \mathrm{Aab}$ & $0,42 \mathrm{Aa}$ & $0,39 \mathrm{ABa}$ & $0,39 \mathrm{Aba}$ & $0,41 \mathrm{Aa}$ \\
\hline Prof/Trat & CCCG & $\mathrm{CCC}$ & $\mathrm{CMCG}$ & $\mathrm{CMC}$ & $\mathrm{APCG}$ & $\mathrm{APC}$ & PDCG \\
\hline $0-5 \mathrm{~cm}$ & $0,21 \mathrm{Aa}$ & $0,19 \mathrm{Aa}$ & $0,13 \mathrm{Ba}$ & $0,11 \mathrm{Ba}$ & $0,09 \mathrm{Bb}$ & $0,09 \mathrm{Ba}$ & $0,09 \mathrm{Ba}$ \\
$5-15 \mathrm{~cm}$ & $0,07 \mathrm{Ab}$ & $0,09 \mathrm{Ab}$ & $0,08 \mathrm{Ab}$ & $0,10 \mathrm{Aa}$ & $0,07 \mathrm{Ab}$ & $0,09 \mathrm{Aa}$ & $0,08 \mathrm{Aa}$ \\
$15-30 \mathrm{~cm}$ & $0,06 \mathrm{Bb}$ & $0,10 \mathrm{ABb}$ & $0,11 \mathrm{ABab}$ & $0,10 \mathrm{ABa}$ & $0,14 \mathrm{Aa}$ & $0,08 \mathrm{ABa}$ & $0,11 \mathrm{Aba}$ \\
\hline Prof/Trat & CCCG & $\mathrm{CCC}$ & $\mathrm{CMCG}$ & $\mathrm{CMC}$ & $\mathrm{APCG}$ & $\mathrm{APC}$ & PDCG \\
\hline $0-5 \mathrm{~cm}$ & $0,25 \mathrm{Cb}$ & $0,29 \mathrm{ABa}$ & $0,28 \mathrm{BCa}$ & $0,31 \mathrm{ABa}$ & $0,31 \mathrm{ABa}$ & $0,32 \mathrm{Aa}$ & $0,31 \mathrm{ABa}$ \\
$5-15 \mathrm{~cm}$ & $0,29 \mathrm{Aa}$ & $0,31 \mathrm{Aa}$ & $0,29 \mathrm{Aa}$ & $0,31 \mathrm{Aa}$ & $0,30 \mathrm{Aa}$ & $0,30 \mathrm{Aa}$ & $0,30 \mathrm{Aa}$ \\
$15-30 \mathrm{~cm}$ & $0,29 \mathrm{Aa}$ & $0,31 \mathrm{Aa}$ & $0,31 \mathrm{Aa}$ & $0,32 \mathrm{Aa}$ & $0,26 \mathrm{Bb}$ & $0,31 \mathrm{Aa}$ & $0,29 \mathrm{Aa}$ \\
\hline Prof/Trat & $\mathrm{CCCG}$ & $\mathrm{CCC}$ & $\mathrm{CMCG}$ & $\mathrm{CMC}$ & $\mathrm{APCG}$ & $\mathrm{APC}$ & PDCG \\
\hline $0-5 \mathrm{~cm}$ & $1,24 \mathrm{Ba}$ & $1,23 \mathrm{Ba}$ & $1,45 \mathrm{Aa}$ & $1,47 \mathrm{Aa}$ & $1,40 \mathrm{ABa}$ & $1,39 \mathrm{ABa}$ & $1,51 \mathrm{Aa}$ \\
$5-15 \mathrm{~cm}$ & $1,15 \mathrm{Aa}$ & $1,19 \mathrm{Aab}$ & $1,10 \mathrm{Ab}$ & $1,19 \mathrm{Ab}$ & $1,07 \mathrm{Ab}$ & $1,01 \mathrm{Ab}$ & $1,19 \mathrm{Ab}$ \\
$15-30 \mathrm{~cm}$ & $1,12 \mathrm{Aa}$ & $1,07 \mathrm{ABb}$ & $1,08 \mathrm{ABb}$ & $0,90 \mathrm{Bc}$ & $0,94 \mathrm{ABb}$ & $1,01 \mathrm{ABb}$ & $0,95 \mathrm{ABc}$ \\
\hline
\end{tabular}

Letras distintas maiúsculas na linha e minúsculas na coluna diferem entre si pelo teste de Tukey, a 0,05 de probabilidade. CCCG representa cultivo convencional + calcário + gesso; CCC, cultivo convencional + calcário; APCG, ausência de preparo + calcário + gesso; CMC, cultivo mínimo + calcário; CMCG, cultivo mínimo + calcário + gesso; APC, ausência de preparo + calcário; e PDCG, plantio direto + calcário + gesso. 
15-30 cm), exceto o Ca e o $\mathrm{Mg}$ nos tratamentos em que a aplicação de calcário e gesso foi incorporada (sistema de cultivo convencional). Nesses tratamentos (CCCG e CCC), a diferença tende a ocorrer na última camada $(15-30 \mathrm{~cm})$. A maior disponibilidade de $\mathrm{P}$ nos tratamentos sem revolvimento do solo (sistemas conservacionistas) devese ao seu acúmulo superficial por ser um elemento de baixa mobilidade. Assim, com o acúmulo, é de se esperar uma diminuição no processo de fixação. Esses resultados são corroborados por pesquisas realizadas por Caires et al. (1998), Wiethölter (2000), Siqueira Neto (2006) e Souza et al. (2007).
Os teores de $\mathrm{N}$ são os que apresentaram menor tendência de variação com a profundidade, o que sugere que o $\mathrm{N}$ mineral está contribuindo com o $\mathrm{N}$ total, a ponto desse não acompanhar do decréscimo de COT com a profundidade (Corrêa, 1989). Observa-se que a relação C/ $\mathrm{N}$ pouco varia com a profundidade.

Como todos os tratamentos receberam aplicação de calcário, os teores de $\mathrm{Al}$ foram anulados até $15 \mathrm{~cm}$ de profundidade quando a calagem foi incorporada (cultivo convencional) independente da aplicação de gesso agrícola. Nos sistemas de manejo conservacionistas, o Al foi totalmente corrigido apenas na primeira camada $(0-5 \mathrm{~cm})$.

Tabela 3 - Valores de carbono orgânico total, fósforo, cálcio e magnésio sob diferentes sistemas de cultivo do solo, em diferentes profundidades.

\begin{tabular}{|c|c|c|c|c|c|c|c|}
\hline Prof/Trat & CCCG & $\mathrm{CCC}$ & CMCG & CMC & APCG & APC & PDCG \\
\hline $0-5 \mathrm{~cm}$ & 2,09 $\mathrm{BCa}$ & $1,90 \mathrm{Ca}$ & $2,24 \mathrm{ABCa}$ & $2,31 \mathrm{ABa}$ & $2,46 \mathrm{ABa}$ & $2,55 \mathrm{Aa}$ & $2,44 \mathrm{ABa}$ \\
\hline $5-15 \mathrm{~cm}$ & $1,67 \mathrm{ABb}$ & $1,68 \mathrm{ABab}$ & $1,81 \mathrm{ABb}$ & $1,71 \mathrm{ABb}$ & $1,59 \mathrm{Bb}$ & $2,08 \mathrm{Ab}$ & $2,07 \mathrm{Ab}$ \\
\hline $15-30 \mathrm{~cm}$ & $1,65 \mathrm{Ab}$ & $1,40 \mathrm{Ab}$ & $1,49 \mathrm{Ab}$ & $1,34 \mathrm{Ac}$ & $1,56 \mathrm{Ab}$ & $1,75 \mathrm{Ac}$ & $1,66 \mathrm{Ac}$ \\
\hline Prof/Trat & CCCG & $\mathrm{CCC}$ & CMCG & CMC & APCG & APC & PDCG \\
\hline $0-5 \mathrm{~cm}$ & $11,98 \mathrm{Da}$ & $11,85 \mathrm{Da}$ & $43,05 \mathrm{Aa}$ & $31,38 \mathrm{Ba}$ & $44,13 \mathrm{Aa}$ & $48,10 \mathrm{Aa}$ & $22,53 \mathrm{Ca}$ \\
\hline $5-15 \mathrm{~cm}$ & 14,08 CDa & $14,58 \mathrm{CDa}$ & $18,33 \mathrm{BCb}$ & $27,80 \mathrm{Aa}$ & $22,93 \mathrm{ABb}$ & $9,48 \mathrm{Db}$ & $6,80 \mathrm{Db}$ \\
\hline $15-30 \mathrm{~cm}$ & $3,65 \mathrm{Ab}$ & $2,20 \mathrm{Ab}$ & $3,43 \mathrm{Ac}$ & $4,68 \mathrm{Ab}$ & $2,98 \mathrm{Ac}$ & $4,45 \mathrm{Ab}$ & $4,25 \mathrm{Ab}$ \\
\hline Prof/Trat & CCCG & $\mathrm{CCC}$ & CMCG & CMC & APCG & APC & PDCG \\
\hline $0-5 \mathrm{~cm}$ & $2,34 \mathrm{Ca}$ & $2,20 \mathrm{BCa}$ & $2,84 \mathrm{Aa}$ & $2,84 \mathrm{Aa}$ & $3,00 \mathrm{Aa}$ & $2,78 \mathrm{ABa}$ & $2,98 \mathrm{Aa}$ \\
\hline $5-15 \mathrm{~cm}$ & $2,38 \mathrm{Aa}$ & $1,35 \mathrm{Bb}$ & $1,55 \mathrm{Bb}$ & $1,55 \mathrm{Bb}$ & $1,60 \mathrm{Bb}$ & $0,65 \mathrm{Cb}$ & $1,78 \mathrm{Bb}$ \\
\hline $15-30 \mathrm{~cm}$ & $1,01 \mathrm{Ab}$ & $0,75 \mathrm{ABc}$ & $0,45 \mathrm{Bc}$ & $0,45 \mathrm{Bc}$ & $0,40 \mathrm{Bc}$ & $0,33 \mathrm{Bb}$ & $0,50 \mathrm{Bc}$ \\
\hline Prof/Trat & CCCG & $\mathrm{CCC}$ & CMCG & CMC & APCG & APC & PDCG \\
\hline $0-5 \mathrm{~cm}$ & $0,88 \mathrm{Da}$ & $1,08 \mathrm{CDa}$ & $0,78 \mathrm{Da}$ & $1,65 \mathrm{Aa}$ & $1,25 \mathrm{BCa}$ & $1,55 \mathrm{ABa}$ & $0,78 \mathrm{Da}$ \\
\hline $5-15 \mathrm{~cm}$ & $0,75 \mathrm{ABa}$ & $0,85 \mathrm{Aa}$ & $0,48 \mathrm{BCb}$ & $0,78 \mathrm{ABb}$ & $0,43 \mathrm{Cb}$ & $0,30 \mathrm{Cb}$ & $0,55 \mathrm{ABCab}$ \\
\hline $15-30 \mathrm{~cm}$ & $0,25 \mathrm{Ab}$ & $0,40 \mathrm{Ab}$ & $0,15 \mathrm{Ac}$ & $0,35 \mathrm{Ac}$ & $0,18 \mathrm{Ab}$ & $0,15 \mathrm{Ab}$ & $0,30 \mathrm{Ab}$ \\
\hline
\end{tabular}

Letras distintas maiúsculas na linha e minúsculas na coluna diferem entre si pelo teste de Tukey, a 0,05 de probabilidade. CCCG representa cultivo convencional + calcário + gesso; CCC, cultivo convencional + calcário; APCG, ausência de preparo + calcário + gesso; CMC, cultivo mínimo + calcário; CMCG, cultivo mínimo + calcário + gesso; APC, ausência de preparo + calcário; e PDCG, plantio direto + calcário + gesso. 
Tabela 4 - Valores de potássio, nitrogênio e alumínio sob diferentes sistemas de cultivo do solo, em diferentes profundidades.

\begin{tabular}{|c|c|c|c|c|c|c|c|}
\hline Prof/Trat & CCCG & $\mathrm{CCC}$ & CMCG & CMC & APCG & APC & PDCG \\
\hline $0-5 \mathrm{~cm}$ & $34,75 \mathrm{Ca}$ & $41,75 \mathrm{BCa}$ & $77,50 \mathrm{Aa}$ & $69,75 \mathrm{Aa}$ & $67,75 \mathrm{Aa}$ & $85,00 \mathrm{Aa}$ & $64,25 \mathrm{ABa}$ \\
\hline $5-15 \mathrm{~cm}$ & $14,25 \mathrm{Ab}$ & $14,25 \mathrm{Ab}$ & $23,75 \mathrm{Ab}$ & $23,25 \mathrm{Ab}$ & $19,00 \mathrm{Ab}$ & $19,25 \mathrm{Ab}$ & $18,50 \mathrm{Ab}$ \\
\hline $15-30 \mathrm{~cm}$ & $9,75 \mathrm{Ab}$ & $15,50 \mathrm{Ab}$ & $20,00 \mathrm{Ab}$ & $15,75 \mathrm{Ab}$ & $10,50 \mathrm{Ab}$ & $15,50 \mathrm{Ab}$ & $16,75 \mathrm{Ab}$ \\
\hline Prof/Trat & CCCG & $\mathrm{CCC}$ & CMCG & CMC & APCG & APC & PDCG \\
\hline $0-5 \mathrm{~cm}$ & 1,05 Aa & 1,05 Aa & 1,05 Aa & 1,05 Aa & 1,05 Aa & 1,05 Aa & 1,05 Aa \\
\hline $5-15 \mathrm{~cm}$ & 1,05 Aa & 1,04 Aa & $0,79 \mathrm{Bb}$ & 1,05 Aa & 0,96 Аа & 1,03 Aa & 1,06 Aa \\
\hline $15-30 \mathrm{~cm}$ & $0,70 \mathrm{Bb}$ & $0,70 \mathrm{Bb}$ & $0,70 \mathrm{Bb}$ & $0,70 \mathrm{Bb}$ & 1,03 Aa & $1,00 \mathrm{Aa}$ & $0,70 \mathrm{Bb}$ \\
\hline Prof/Trat & CCCG & $\mathrm{CCC}$ & CMCG & CMC & APCG & APC & PDCG \\
\hline $0-5 \mathrm{~cm}$ & $0,00 \mathrm{Ab}$ & $0,00 \mathrm{Ab}$ & $0,03 \mathrm{Ac}$ & $0,00 \mathrm{Ac}$ & $0,00 \mathrm{Ab}$ & $0,00 \mathrm{Ab}$ & $0,00 \mathrm{Ab}$ \\
\hline $5-15 \mathrm{~cm}$ & $0,00 \mathrm{Cb}$ & $0,00 \mathrm{Cb}$ & $0,33 \mathrm{Bb}$ & $0,28 \mathrm{Bb}$ & $0,10 \mathrm{Cb}$ & $0,80 \mathrm{Aa}$ & $0,08 \mathrm{Cb}$ \\
\hline $15-30 \mathrm{~cm}$ & $0,50 \mathrm{BCa}$ & $0,53 \mathrm{BCa}$ & $0,65 \mathrm{ABa}$ & $0,43 \mathrm{Ca}$ & $0,55 \mathrm{ABCa}$ & $0,70 \mathrm{Aa}$ & $0,43 \mathrm{Ca}$ \\
\hline
\end{tabular}

Letras distintas maiúsculas na linha e minúsculas na coluna diferem entre si pelo teste de Tukey, a 0,05 de probabilidade. CCCG representa cultivo convencional + calcário + gesso; CCC, cultivo convencional + calcário; APCG, ausência de preparo + calcário + gesso; CMC, cultivo mínimo + calcário; CMCG, cultivo mínimo + calcário + gesso; APC, ausência de preparo + calcário; e PDCG, plantio direto + calcário + gesso.

\section{CONCLUSÕES}

Em todos os sistemas de cultivo pesquisados ocorre compactação subsuperficial, indicada pela redução da relação macro/microporos, acompanhada pelo aumento da densidade do solo.

Devido à compactação, a estabilidade de agregados (diâmetro médio geométrico) diminui com a profundidade.

Na profundidade de $0-5 \mathrm{~cm}$, o diâmetro médio geométrico (DMG) é maior em todos os tratamentos, com destaque para os sistemas conservacionistas devido à ausência de revolvimento do solo.

Quanto aos atributos químicos, as diferenças significativas entre os tratamentos conservacionistas e convencional ocorrem apenas nos primeiros $5 \mathrm{~cm}$ do solo, somente para os elementos $\mathrm{P}, \mathrm{Ca}$ e $\mathrm{K}$.

A correção do Al é mais eficiente no sistema convencional, independente da aplicação de gesso agrícola, devido à incorporação desse corretivo ao solo.

\section{REFERÊNCIAS BIBLIOGRÁFICAS}

ALBUQUERQUE, J.A.; REINERT, D.J.; FIORIN, J.E.; RUEDELL, J.; PETRERE, C.; FONTINELLI, F. Rotação de culturas e sistemas de manejo do solo: efeito sobre a forma da estrutura do solo ao final de sete anos. Revista Brasileira de Ciência do Solo, Campinas, v.19, p.115-119, 1995.
ANDREOLA, F.; COSTA, L.M.; OLSZEVSKI, N. Influência da cobertura vegetal de inverno e da adubação orgânica e, ou, mineral sobre as propriedades físicas de uma Terra Roxa Estruturada. Revista Brasileira de Ciência do Solo, Campinas, v.24, p.857865,2000 .

ANJOS, J.T.; UBERTI, A.A.A.; VIZZOTTO, V.J.; LEITE, G.B.; KRIEGER, M. Propriedades físicas em solos sob diferentes sistemas de uso e manejo. Revista Brasileira de Ciência do Solo, Campinas, v.18, p.139145, 1994.

CAIRES, E.F.; CHUEIRI, W.A.; MADRUGA, E.F.; FIGUEIREDO, A. Alterações de características químicas do solo e resposta da soja ao calcário e gesso aplicados na superfície em sistema de cultivo sem preparo do solo. Revista Brasileira de Ciência do Solo, Campinas, v.22, p.27-34, 1998.

CANTARELLA, H. Nitrogênio. In: NOVAIS, R.F.; ALVAREZ, V.H.; BARROS, N.F. de; FONTES, R.L.F.; CANTARUTTI, R.B.; NEVES, J.C.L. Fertilidade do solo. Viçosa, MG: Sociedade Brasileira de Ciência do Solo, 2007. 1017p. 
COMISSÃO DE FERTILIDADE DO SOLO DO ESTADO DE MINAS GERAIS. Recomendações para o uso de corretivos e fertilizantes em Minas Gerais: $5^{\text {a }}$ aproximação. Viçosa, MG, 1999. 359p.

CORRÊA, G.F. Modelo de evolução e mineralogia da fração argila de solos do planalto de Viçosa, MG. 1984. 87f. Dissertação (Mes-trado)-Universidade Federal de Viçosa, Viçosa, 1984.

CORRÊA, G.F. Les microrelief "murundus" et leur environnement pédologique dans l'ouest du Minas Gerais, région du plateau central brésilien. 1989. 144f. Tese (Doutorado)-Université de Nancy I, Vandoeuvreles-Nancy, 1989.

CRUZ, A.C.R.; PAULETTO, E.A.; FLORES, C.A.; SILVA, J.B. Atributos físicos e carbono orgânico de um Argissolo Vermelho sob sistemas de manejo. Revista Brasileira de Ciência do Solo, Campinas, v.27, p.11051112, 2003.

\section{EMPRESA BRASILEIRA DE PESQUISA}

AGROPECUÁRIA. Centro Nacional de Pesquisa de Solos. Manual de métodos de análise de solo. 2.ed. Rio de Janeiro, 1997. 212p.

EMPRESA BRASILEIRA DE PESQUISA AGROPECUÁRIA. Serviço Nacional de Levantamento e Conservação dos Solos. Levantamento de média intensidade dos solos e avaliação da aptidão agrícola das terras do Triângulo Mineiro. Rio de Janeiro, 1982. 526p. (Boletim técnico, 1).

FERREIRA, D.F. Análises estatísticas por meio do Sisvar para o Windows versão 4.0. In: REUNIÃO ANUAL DA REGIÃO BRASILEIRA DA SOCIEDADE

INTERNACIONAL DE BIOMETRIA, 45., 2000, São Carlos. Anais... São Carlos: UFSCar, 2000. p.255-258.

MARIA, I.C. de; NNABUDE, P.C.; CASTRO, O.M. Long-term tillage and crop rotation effects on soil chemical properties of a Rhodic Ferralsol in southern Brazil. Soil Tillage Research, Amsterdam, v.51, p.71-79, 1999.
MIYASAKA, S.; CAMARGO, A.P.; INFORZATO, R. Efeito da cobertura e da incorporação ao solo imediatamente antes do plantio de diferentes formas de matéria orgânica não decomposta, na cultura do feijoeiro. Bragantia, Campinas, v.24, n.32, p.349-69, 1996.

SÁ, J.C.M. Manejo da fertilidade do solo no plantio direto. Castro: Fundação ABC, 1993. 96p.

SILVEIRA, P.M.; STONE, L.F. Teores de nutrientes e de matéria orgânica afetados pela rotação de culturas em sistema de preparo de solo. Revista Brasileira de Ciência do Solo, Campinas, v.25, p.387-394, 2001.

SIQUEIRA NETO, M. Estoque de carbono e nitrogênio no solo com diferentes usos no Cerrado em Rio Verde (GO). 2006. 162p. Tese (Doutorado em Energia Nuclear na Agricultura)-Escola Superior de Agricultura Luiz de Queiroz, Universidade de São Paulo, Piracicaba, 2006.

SOUZA, C.H.E.; EZEQUIEL JÚNIOR, S.M.; FIGUEIREDO, C.C.; COSER, T.; TORRES, A.L. Alterações de propriedades químicas de solo sob sistemas de manejo no cerrado. In: CONGRESSO BRASILEIRO DE CIÊNCIAS DO SOLO, 31., 2007. Anais... 2007.

TEDESCO, M.J.; VOLKWEIIS, S.J.; BOHNEN, H. Análises de solo, plantas e outros materiais. Porto Alegre: UFRGS, 1985. 188p. (Boletim técnico, 5).

WIETHOLTER, S. Manejo da fertilidade do solo no sistema plantio direto: experiência nos estados do Rio Grande do Sul e Santa Catarina. In: REUNIÃO BRASILEIRA DE FERTILIDADE DO SOLO E NUTRIÇÃO DE PLANTAS, 25.; REUNIÃO BRASILEIRA SOBRE MICORRIZAS, 8.; SIMPÓSIO BRASILEIRO DE MICROBIOLOGIA DO SOLO, 6.; REUNIÃO BRASILEIRA DE BIOLOGIA DO SOLO, 3., 2000, Santa Maria. Anais... Santa Maria: UFSM, 2000. CD-ROM.

YEOMANS, J.C.; BREMNER, J.M. A rapid and precise method for routine determination of organic carbon in soil. Communication Soil Science Plant Analytical, v.19, p.1467-1476, 1988. 